\title{
Juvenile Primary Fibromyalgia Syndrome
}

National Cancer Institute

\section{Source}

National Cancer Institute. Juvenile Primary Fibromyalgia Syndrome. NCI Thesaurus. Code C117297.

Chronic, diffuse, non-inflammatory musculoskeletal pain disorder associated with fatigue and sleep disturbance that can occur in childhood and adolescence. Tender points are not necessary to make the diagnosis, but if present may be less numerous than found in adults. 